\title{
Hypotensive effect of resistance exercises performed with different intensities in hypertensive elderly women
}

\author{
Nívea Maria de Oliveira Jacques ${ }^{1}$, Felipe J Aidar ${ }^{2}$, Luis Leitão ${ }^{3,4}$, Mário Espada ${ }^{3,4}$, Aldo M Costa ${ }^{5}$ and Ana Pereira ${ }^{3,4}$ \\ ${ }^{1}$ Departament of Physical Education and Sport, Estadual University of Montes Claros, Minas Gerais, Brazil \\ ${ }^{2}$ Department of Physical Education, Federal University of Sergipe, São Cristóvão, Brazil \\ ${ }^{3}$ Superior School of Education of Polytechnic Institute of Setubal, Portugal; Research Center in Education (CIEF) of Polytechnic Institute of Setubal Portugal \\ ${ }^{4}$ Quality of Life Research Center, Polytechnic Institute of Santarem, Portugal \\ ${ }^{5}$ Department of Sports Sciences, University of Beira Interior, Covilhã, Portugal, Research Center in Sports Sciences, Health Sciences and Human Development, \\ CIDESD, Vila Real, Portugal, Health Sciences Research Center, CICS-UBI, Covilhã, Portugal
}

\begin{abstract}
Systemic arterial hypertension represents one of the principal risk factors for the increase of cardiovascular diseases, especially in the elderly people. On post-exercise, blood pressure responses (BP) may decrease and this effect have a relevant clinical importance. However, further studies are needed to better understand the effects of different intensities and exercise modes. The aim of this study was to analyse the hypotensive effect of resistance exercises on BP of controlled hypertensive women performed at different intensities. The study sample was composed by 13 hypertensive elderly women ( $65.5 \pm 4.2$ years), submitted to two different training intensities: $50 \%$ and $70 \%$ of $10 \mathrm{RM}$. After the resistant exercise systolic and diastolic blood pressure were measured during 1 hour and every 10 minutes. Significant differences were found only in diastolic blood pressure (DBP) measured at 10 ( $p=0.034), 20$ ( $p=0.042), 30$ ( $p=0.031$ ), 45 ( $p=0.035)$ and 60 minutes ( $p=0.017$ ) after the effort. Our data suggest that resistance exercise does not result in $\mathrm{PEH}$ in hypertensive elderly women without significant effects on diastolic blood pressure at different intensities.
\end{abstract}

\section{Introduction}

In Brazil, one risk factor for cardiovascular disease is hypertension and is important to reduce the main values [1]. According to the world health organization (WHO), the number of hypertensive patients is of approximately one billion, with 7.1 million of deaths per year. Systemic arterial hypertension (HBP) is characterized by a chronic increase in systolic and diastolic blood pressure above acceptable levels for human health. Besides, is a multifactorial disease with high prevalence in the elderly population. It reaches a large part of the adult population worldwide, making it a cause for public health.

Pharmacological treatment is indicated for moderate and severe hypertensive patients, and for those with risk factors for cardiovascular diseases and/or major target organ injury. However, few hypertensive patients achieve optimal control pressure with a single therapeutic agent and, often, combined therapy is necessary, especially in elderly people with relevant co-morbidities [2]. Although, drug therapy is effective in reducing blood pressure values, morbidity and mortality but has a high cost [3]. Resistance exercise (RE) is recommended for that disease. However, the literature lacks studies that demonstrate the acute hypotensive effect of resistent exercise (RE) in hypertensive elderly subjects. Most of the studies [4,5] demonstrates the onset of BP decrease after performance of aerobic exercises, although, there are few studies analysing the effect of resisted exercise in post-exercise hypotension [6] specifically in hypertensive elderly women [7]. Besides, RE has been recommended as part of the non-pharmacological treatment of HBP because it is possible to observe a hypotensive effect, with the decreased blood pressure levels below the rest values after effort. Nevertheless, there are many positive benefits, as better quality of life and function provided by the physical training, which reduces the deleterious physiological alterations with ageing. However, there are few studies that address different intensities and their effect on HBP. Thus, is very important to understand how is the interference is after the exercise period. Thus, this study aimed to analyse the effect of post -exercise hypotension after resistance exercises performed at different intensities in controlled hypertensive elderly women.

\section{Methods}

\section{Sample}

The study sample consisted of Brazilian women aged between 60 and 76 years (Table 1) all with systemic arterial hypertension. The sample consisted of 13 elderly women (GI) with clinically diagnosed arterial hypertension and controlled by anti-hypertension medication. All volunteers are participants of the Oriented Walk Program, an institutionalized project of the Physical Education course of the State University of Montes Claros, but without previous experience in resistance exercise. All of them used at least one drug to control systemic arterial hypertension and made two shots of the drug, one in the morning another at night. They were instructed to continue with

*Correspondence to: Aldo M. Costa, Department of Sports Sciences, University of Beira Interior, Covilhã, Portugal, Research Center in Sports Sciences, Health Sciences and Human Development, CIDESD, Vila Real, Portugal, Health Sciences Research Center, CICS-UBI, Covilhã, Portugal, E-mail: anapereiraphd@gmail.com

Key words: post-exercise hypotension, resistance training, elderly, intensity

Received: November 07, 2019; Accepted: November 25, 2019; Published: November 29, 2019 
Table 1. General characteristics of the studied population

\begin{tabular}{|c|c|c|c|c|c|}
\hline & Average & $\begin{array}{c}\text { Standard } \\
\text { deviation }\end{array}$ & Minimun & Maximum \\
\hline \multirow{4}{*}{ GI } & age (years) & 65.5 & 4.2 & 60 & 73 \\
\cline { 2 - 6 } & Body mass (kg) & 71.35 & 7.45 & 61.9 & 89.0 \\
\cline { 2 - 6 } & Heighy (m) & 1.55 & 0.07 & 1.45 & 1.66 \\
\cline { 2 - 6 } & $\mathbf{B M I}\left(\mathbf{k g} / \mathbf{m}^{\mathbf{2}}\right)$ & 29.71 & 3.77 & 24.42 & 36.57 \\
\cline { 2 - 6 } & $\mathbf{S B P}(\mathbf{m m H g})$ & 135.85 & 12.15 & 120 & 150 \\
\cline { 2 - 6 } & $\mathbf{D B P}(\mathbf{m m H g})$ & 88.38 & 11.5 & 70 & 110 \\
\hline
\end{tabular}

GI: Group of intervention elderly women; SBP: sstolic blood pressure; DBP: Diastolic blood pressure; BMI: Body mass index

their medications and to not interrupt the other activities, such as walks and their activities of daily living, such as passing, washing, taking care of the house and cooking.

\section{Methods and procedures}

During the days undergoing the experiment, the patients were instructed to not consume caffeine and alcoholic before 24 hours of the blood and data collection and not practice physical activity before RE sessions. All individuals signed the Free and Informed Consent Form according to resolutions $196 / 96$ and 466/2012 of the National Health Council, which contains the guidelines and norms that regulate research involving human beings, in accordance with the ethical principles contained in the Declaration of Helsinki (1964, reformulated in 1975, 1983, 1989, 1996, 2000 and 2008) of the "World Medical Association". This study is approved by the Ethics Committee under number 31856014.0.0000.5146.

All volunteers were informed of the research objectives and the experimental procedures to which they were subjected. Also, they were informed about the possible risks and discomfort that they could feel in performing the RE. Although, all benefits of the present study were also described. They were informed about the confidentiality of the study regarding their identities and in addition, informed that they could interrupt their participation at any time without providing the reason or reason for their interruption. All the women were submitted to a clinical evaluation to enter the engage in the resistance exercise. The exclusion criteria of this research were: a) non-return of the signed Free and Informed Consent Form; b) carry osteo-articular problems that could partially or fully affect the performance of specific movements of the tests; c) have respiratory and/or metabolic problems; d) be a smoker; e) be diabetic; f) present other medical contraindications such as: uncontrolled arterial hypertension, congestive heart failure, recent myocardial infarction, and among other diseases that would compromise cardiovascular responses as sedative medication, confusion or dementia and/or cognitive deficit which could hamper the tests' execution were not selected either. Besides these criteria, the women who presented inappropriate blood pressure response or heart rate and/or ventricular arrhythmias induced by exertion of the previous RE test were excluded from the study. Those women with number of absences higher than $10 \%$ of the proposed period for the training protocol were also excluded from the sample.

For data collection, a meeting was first held of the researcher with individuals of the Oriented Walk Program, Institutionalized Project of the State University of Montes Claros UNIMONTES, in order to clarify the objectives of the study, as well as all the procedures involved. Large numbers of subjects were interested in participating in the study, but did not have the specific conditions for the research.

The physical characteristics of the sample were collected in the physical evaluation room of the Laboratory of Exercise of
UNIMONTES. For body mass measurements, a Toledo digital scale (Toledo, Brazil) was used with a stake meter coupled with a capacity of 0 to $150 \mathrm{~kg}$ and accuracy of $0.05 \mathrm{~kg}$ and the height, was measured with volunteers stepping in the centre of the scale, with its back to scale, in an orthostatic position [8].

Two weeks of adaptation of the RE were performed with all the volunteers of the study in those exercises that composed the training program, in order to minimize risks and have a good reproducibility in the execution of movements.

\section{Test of 10 maximum repetitions (10-RM)}

The subjects performed two weeks of adaptation to the exercises to learn the correct performance technique, normal range of motion and suitable breathing. During this period, the exercises were performed without load. To establish the load percentage of $50 \%$ and $70 \%$, which are objectives of this study, the 10 RM test [9] was applied. To verify the reproducibility of the test, the retest was performed with the volunteers after one week.

To determine the maximum load, three attempts were assigned for each subject with an interval of 5 minutes between them; in subsequent visits, the subjects remained 30 minutes seated in a calm and comfortable place, for further measurement of BP. BP was taken in the left upper limb three times and then we made the average. Subsequently, they performed self-stretching of the main muscles involved in the exercises of the program (15 seconds): lower limbs (quadricipitis and femoral bicipitis), pectoral and posterior trunk. After that, the individuals underwent a warm-up in the cycle ergometer (BM2700 Moviment Tecnology, Brudden, Brazil) during 10 minutes.

The exercise program consisted of three series of 10 repetitions always performed in the following order: leg press, bench press, knee extension with extensor chair, front pull and knee flexion on flexor table (Vitally Start, Brazil). With two minutes interval between sets and the performance velocity of the exercises was of 2:2. In each visit, a draw was made of the percentage of the load that was made on the day. The subjects perform an expiration during the concentric contraction and an inspiration during the eccentric contraction to avoid the Valsalva's manouver. The 10 RM test followed the recommendations [9]. The individual should mobilize a load that prevented him from performing more than $10 \mathrm{MR}$ under maximum voluntary concentric muscle failure regimen. Modified Borg's scale [9] was sued to question on the perceived exertion with the load intensity in each trail. The tests were developed during a single day for the hight exercises, immediately after the adaptation period. In order to reduce the margin of error, the following strategies were adopted: 1) Standardized instructions were offered before the test, so that the evaluated was aware of the entire routine that involved data collection; 2) the evaluation was instructed on the technique of performing the exercise; 3) the evaluator was attentive to the position adopted by the practitioner at the time of the measurement. Small variations in the positioning of the joints involved in the action could recruit other muscles, distancing from the specific focus of the research and enabling erroneous interpretations of the scores obtained could happened; and, 4) a fixed position was stipulated for the foot, thus avoiding differentiation in the angulation of the same individual's ankle in both tests at $50 \%$ and $70 \%$ of 10RM.

\section{Blood pressure measurement}

A Microlife device, model BP 3AC1-1 (manufactured by Microlife/ USA), validated according to the criteria of the British Association of Cardiology for resting measures [10] was used to assess the systolic and 
diastolic blood pressure of the subjects. The first moment was at rest, followed by 10-minute intervals after the end of the resistance training until completion of an hour, performing six moments: 1st, 10th, 20th, 30 th, 45th and 60th minutes, with volunteers sitting comfortably, in a room adjacent to the training hall at 8 a.m. BP was measured by the researcher and 2 academics of the Physical Education course trained in the Laboratory of Exercise with more than 1 year of experience. Room temperature was 24 celsius degrees and humidity was around $56 \%$. The measures followed the recommendations of the VI Brazilian Guidelines on Hypertension [11]. The measurements were always performed by the researcher and in the same period of the day.

The volunteers were instructed to not contract or move the arm, hand and fingers of the upper-limb where the BP sealer was attached. Also, to not talking and to not performing the Valsava Maneuver. Volunteers were also asked to keep their elbow semi-flexed, in order to approach the chest hand and prevent the recorded values from being contaminated by gravitational action if the arm remained extended [12].

\section{Statistical treatment}

The data were analysed with the statistical program SPSS, version 17.0 (SPSS Inc., Chicago, IL, USA), with significance level of 5\%. Descriptive statistics were applied with central trend measurements (mean \pm standard deviation) and the variables normality was still verified using the Shapiro Wilk test, in view of the sample size. The sphericity was ensured through the Mauchly test. To verify variations in the domains, a MANOVA test, Post Hoc de Bonferroni was performed. $\mathrm{BP}$ was compared at rest, and in the $1 \mathrm{st}, 10 \mathrm{th}, 20 \mathrm{th}, 30 \mathrm{th}, 45^{\circ}$ and $60^{\circ}$ minutes, at different intensities (50\% and $70 \%)$. The significance level adopted was $\mathrm{p} \leq 0.05$. To verify the size of the effect, the values of eta $(\mu \mathrm{p} 2)$ were used, adopting the low effect values $(0.1$ and 0.24$)$, mean effect (0.25 and 0.39) f2 test and high effect (greater than 0.40$)$.

\section{Results}

Table 2 shows the values related to $\mathrm{BP}$ at rest, pre-test, rest and all moments related to the 1st, 10th, 20th, 30th, 45th and 60th minutes after the intervention.

According to the results, the SBP values at all times did not present a significant difference in relation to rest and the intensities of $50 \%$ and $70 \%$ of $10 \mathrm{RM}$. Although, there was no significant difference in DBP at

Table 2. Description of the mean values, standard deviation and $p$ value for the systolic and diastolic blood pressure in each evaluation sequence in the low and high intensity exercise

\begin{tabular}{|c|c|c|c|c|c|}
\hline \multirow{2}{*}{ Sequences } & \multicolumn{3}{|c|}{ GI $(N=13)$} & \multirow{2}{*}{$\mathbf{P}$} & \multirow{2}{*}{$\mu_{\mathrm{p}} 2$} \\
\hline & Rest & $50 \%$ & $70 \%$ & & \\
\hline Rest (SBP) & $130.85 \pm 9.48$ & $130.47 \pm 6.654$ & $129.52 \pm 11.07$ & 0.135 & 0.042 \\
\hline $1 \min (\mathrm{SBP})$ & $132.69 \pm 9.10$ & $138.77 \pm 12.76$ & $142.54 \pm 14.13$ & 0.062 & 0.096 \\
\hline $10 \min (\mathrm{SBP})$ & $132.62 \pm 8.20$ & $134.23 \pm 12.11$ & $139.46 \pm 13.85$ & 0.077 & 0.051 \\
\hline $20 \min (\mathrm{SBP})$ & $132.54 \pm 9.32$ & $133.00 \pm 9.72$ & $138.38 \pm 12.48$ & 0.067 & 0.058 \\
\hline $30 \mathrm{~min}(\mathrm{SBP})$ & $131.54 \pm 9.45$ & $134.54 \pm 11.98$ & $137.92 \pm 9.97$ & 0.087 & 0.079 \\
\hline $45 \min (\mathrm{SBP})$ & $131.31 \pm 8.91$ & $132.85 \pm 11.06$ & $133.31 \pm 10.19$ & 0.090 & 0.054 \\
\hline $60 \min (\mathrm{SBP})$ & $131.08 \pm 8.98$ & $131.77 \pm 10.22$ & $132.38 \pm 9.22$ & 0.123 & 0.048 \\
\hline Rest (DBP) & $82.62 \pm 4.49$ & $84.54 \pm 11.98$ & $79.65 \pm 5.69 *$ & 0.040 & 0.158 \\
\hline $1 \min (\mathrm{DBP})$ & $87.62 \pm 6.49$ & $84.08 \pm 7.85$ & $88.77 \pm 8.63$ & 0.067 & 0.121 \\
\hline $10 \min (\mathrm{DBP})$ & $87.92 \pm 6.22$ & $83.46 \pm 8.23 *$ & $85.77 \pm 9.10$ & 0.034 & 0.166 \\
\hline $20 \min (D B P)$ & $87.77 \pm 6.72$ & $82.54 \pm 8.46^{*}$ & $85.31 \pm 6.32$ & 0.042 & 0.114 \\
\hline $30 \min (\mathrm{DBP})$ & $87.23 \pm 7.14$ & $80.23 \pm 8.41^{*}$ & $85.08 \pm 5.19$ & 0.031 & 0.167 \\
\hline $45 \min (\mathrm{DBP})$ & $86.77 \pm 6.82$ & $80.31 \pm 8.27 *$ & $83.54 \pm 6.11$ & 0.035 & 0.158 \\
\hline $60 \mathrm{~min}(\mathrm{DBP})$ & $86.54 \pm 6.78$ & $79.46 \pm 7.17 *$ & $83.15 \pm 6.04$ & 0.017 & 0.299 \\
\hline
\end{tabular}

" $\mathrm{p} \leq 0.05$ (MANOVA, and Post Hoc Bonferroni); min: Minute
1 minute after the performance of the resistance exercises. However, there were significant differences in DBP measured at $10^{\prime}(\mathrm{p}=0.034)$, $20^{\prime}(\mathrm{p}=0.042), 30^{\prime}(\mathrm{p}=0.031), 45^{\prime}(\mathrm{p}=0.035)$ and 60' $(\mathrm{p}=0.017)$. This difference was detected in comparation to the rest value, with no difference between.

\section{Discussion}

In the present study, differences were observed after 10 minutes and the lower value occurred in the 60 minutes in relation to DBP.

Our results are divergent observed an absence of post-exercise BP drop [13,14], while other author [15] found reductions up to $40 \mathrm{mmHg}$. Similarly, the duration of post-exercise hypotension ranges from 60 minutes [16] to more than 13 hours [17]. According to several authors $[18,19]$ verified a reduction of SBP and DBP one hour and 10 hours after performing the exercise session in young hypertensive and sedentary subjects. These disparities suggest that some factors may be influencing the decreases in the BP as pharmacological treatment. Identifying these factors have a clinical importance because can help professionals in the prescription of physical exercises in the non-pharmacological treatment of HBP, in this specific study population.

The duration of the exercise session has been suggested as one of the factors that influences post-exercise hypotension. A 10 minutes sets of treadmill walking were observed and did not change BP behaviour in the post-exercise period in normotensive individuals [20]. In addition, in hypertensive individuals, the same protocol already caused a significant decrease in BP and the magnitude of this change expanded when a new exercise set were added to the training session. In another study conducted in spontaneously hypertensive rats [21] found that the pressure reduction after the performance of 40 minutes of exercise on the treadmill was greater and longer than the reduction after 20 minutes of that same exercise. On the other hand, the majority of the studies $[4,5]$ showed a decrease in BP after aerobic exercises were performed, which were not the object of study in this study. Besides, reduced resting BP after RE was observed in several studies [7,22-30] with different protocols and subjects and only a few ones did not observe significant changes in SBP and DBP [31-34]. These results suggest that resistance training may also have a hypotensive effect on the elderly, which corroborate the results of the present study. However, the magnitude of BP modification was different between studies, which suggests that factors related to the characteristics of the studied population, has psychosomatic factors such as fear, anxiety and personality or the training protocol used may have influenced this magnitude. Also, because we used only elderly women and no studies have analysed the effects of RE in hypertensive elderly subjects with different intensities.

However, it is important to emphasize that, in the most of the studies that showed lower BP, the sample also included normotensive individuals, unlike this study that was conducted only with controlled hypertensive elderly women. In addition, in one of these studies, aerobic training was performed concomitantly with the resistance exercise, so the hypotensive effect may be due to aerobic stimulation [26] or both. In other studies, volunteer individuals also had other concomitant metabolic diseases, and these diseases may also have influenced the results $[23,25]$. Finally, in the only study involving only hypertensive elderly [29], patients were using different antihypertensive medications, as well as the present study, which did not allow to determining the isolated effect of training resistance on BP. In studies involving only normotensive individuals, few studies did not see a reduction in clinical $\mathrm{BP}[33,34]$ while others found a drop in this pressure $[7,22,24,25,27,28]$. 
These results suggest that resistance training is effective to reduce BP for normotensive elderly, but its effect on hypertensive patients still needs to be better understood. In the present study, according to the results of SBP, there was no significant difference in relation to rest values comparing to both intensities of $50 \%$ and $70 \%$ of $10 \mathrm{RM}$.

Regarding the effect of resistance training intensity [28] demonstrated, in normotensive elderly, that training performed in lower intensity (between 55 to $65 \%$ of $1 \mathrm{RM}$ ) was able to reduce both SBP and DBP, while resistance training performed with greater intensity (between 75 to $85 \%$ of $1 \mathrm{RM}$ ) only decreased SBP. In fact, other studies conducted with high intensity ( $75 \%$ of $1 \mathrm{RM})$ also observed a reduction in SBP, or even no hypotensive effect [34]. In general, studies involving exercises with the intensities used to develop localized muscle endurance, i.e., low intensity (between 50 to $65 \%$ of $1 \mathrm{RM}$ to $15 \mathrm{RM}$ ), found a decrease in both SBP and DBP $[7,24]$. On the other hand [27] found that resistance exercises performed with high intensity (8 RM) were able to reduce DBP, but had no effect on SBP. Thus, most of the data allows us to assume that resistance training performed with lower intensity would be more recommended in order to promote the reduction of resting BP; however, there are still controversies. Regarding, to the main adaptations associated to the RE, this type of training can promote the increases of elastic components, in the arteries due to progressive fragmentation and destruction of cells and fibers. The development of strength also leading to greater vascular function between the responses of the vasoconstrictor and vasodilator agents [35]. Thus, high $\mathrm{BP}$ also can have a genetic and the environment effect, although, aging seems to contribute to the BP increase [4].

Thus, our study demonstrated an improvement with regard to DBP, corroborating in part with the studies and assumed that the intensity of $50 \%$ would be more beneficial to cause greater hypotensive effect of BP after resistance exercise.

\section{Conclusion}

Our results suggest that the hypotensive effect after resistance exercise was more significant after the training protocol with $50 \%$ of $10 \mathrm{RM}$ in SBP.

\section{Funding}

This research received no specific grant from public, commercial, or not-for-profit sector. It was fully author funded.

\section{Declarations}

The authors declare they have no actual or potential competing financial interests.

\section{Authorship and contributorship}

Nívea Maria de Oliveira Jacques and Felipe J. Aidar: Substantial contributions to conception and design, or acquisition of data, or analysis and interpretation of data; Luis Leitão: agreement to act as guarantor of the work; Mário Espada and Aldo M. Costa: drafting the article or revising it critically for important intellectual content; Ana Pereira: Final approval of the version to be published.

\section{References}

1. $7^{\text {th }}$ Brazilian guideline of arterial hypertension: Presentation (2016) Arquivos Brasileiros de Cardiologia, São Paulo.

2. Oparil S (2001) Hipertensão arterial. In: Goldman L, Bennet JC, organizadores. Cecil Tratado de medicina interna. Rio de Janeiro: Guanabara Koogan.
3. Shoji VM, Forjaz CLM (2000) Treinamento físico da hipertensão. Revista da Sociedade de Cardiologia Estado de São Paulo10: 7-14.

4. Cardoso Jr CG, Gomides RS, Queiroz ACC, Pinto LG, Lobo FS, et al. (2010) Acute and chronic effects of aerobic and resistance exercise on ambulatory blood pressure. Clinics 65: 317-325.

5. Senitko AM, Charkoudian N, Halliwil JR (2002) Influence of endurance exercise training status and gender on postexercise hypotension. Journal of Applied Physiology 92: 2368-2374

6. Simão R, Fleck SJ, Polito M, Monteiro W, Farinatti P (2005) Effects of resistance training intensity, volume, and session format on the postexercise hypotensive response. Journal of Strength \& Conditioning Research 19: 853-858

7. Martel GF, Hurlbut DE, Lott ME, Lemmer JT, Ivey FM, et al. (1999) Strength training normalizes resting blood pressure in 65- to 73-year-old men and women with high normal blood pressure. Journal of the American Geriatrics Society 47: 1215-1221.

8. Schünke M, Schulte E, Schumacher U, Voll M, Wesker K (2006) Prometheus Atlas de Anatomia- Anatomia geral e do aparelho locomotor. $1^{\text {a }}$ ed. Rio de Janeiro: Guanabara Koogan.

9. Baechle TR, Groves BR (2000) Treinamento de força muscular: passos para o sucesso. $2^{\mathrm{a}}$ ed: Porto Alegre, Artmed.

10. Cuckson AC, Reinders A, Shabeeh H, Shennan AH, et al. (2002) Validation of the Microlife BP 3BTO-A oscillometric blood pressure monitoring device according to a modified British Hypertension Society protocol. Blood Pressure Monitoring 7: 319324.

11. VI Diretrizes Brasileiras de Hipertensão (2010) Arquivos Brasileiros de Cardiologia 95: 1-51.

12. Soutello AL, Rodrigues RC, Jannuzzi FF, São-João TM (2015) Quality of Life on Arterial Hypertension: Validity of Known Groups of MINICHAL. Arquivos Brasileiros de Cardiologia 104: 299-307.

13. Cléroux J, Kouamé N, Nadeau A, Coulombe D, Lacoucière Y (19929 Baroreflex regulation of forearm vascular resistance after exercise in hypertensive and normotensive humans. American Physiological Society Journal 263: 1523-1531.

14. Cléroux J, Kouamé N, Nadeau A, Coulombe D, Lacourcière Y (1992) After effects of exercise on regional and systemic hemodynamics in hypertension. Hypertension 19: 183-191.

15. Wilcox RG, Bennett T, Brown AM, MacDonald IA (1982) Is exercise good for high blood pressure? British Medical Journal 285: 767-769.

16. Somers J, Coats A, Isea J, Sleight P (1991) Postexercise hypotension is not sustained in normal and hypertensive humans. Hypertension 18: 211-215.

17. Pescatello LS, Fargo AE, Leach CN Jr, Scherzer HH (1991) Short-term effect of dynamic exercise on arterial blood pressure. Circulation 83: 1557-1561.

18. Hardy DO, Tucker LA (1999) The effects of a single bout of strength training on ambulatory blood pressure levels in 24 mildly hypertensive men. American journal of health promotion 13: 69-72.

19. Melo CM, Alencar Filho AC, Tinucci T, Mion D Jr, Forjaz CL (2006) Postexercise hypotension induced by low-intensity resistance exercise in hypertensive women receiving captopril. Blood Pressure Monitoring 11: 183-189.

20. Bennett T, Wilcox RG, MacDonald IA (1984) Post-exercise reduction of blood pressure in hypertensive men is not due to acute impairment of baroreflex function. Clinical Science 67: 97-103.

21. Overton JM, Joyner MJ, Tipton CM (1988) Reductions in blood pressure after acute exercise by hypertensive rats. Journal of Applied Physiology 64: 748-752.

22. Simons R, Andel R (2006) The effects of resistance training and walking on functional fitness in advanced old age. Journal of Aging and Health 18: 91-105.

23. Castaneda C, Layne JE, Munoz-Orians L, Gordon PL (2002) A randomized controlled trial of resistance exercise training to improve glycemic control in older adults with type 2 diabetes. Diabetes Care 25: 2335-2341.

24. Delmonico MJ, Ferrell RE, Meerasahib A, Martel GF, Roth SM, et al. (2005) Blood pressure response to strength training may be influenced by angiotensinogen A- 20C and angiotensin II type I receptor A1166C genotypes in older men and women. Journal of the American Geriatrics Society 53: 204-210.

25. Sallinen J, Fogelholm M, Pakarinen A, Juvonen T, Volek JS, et al. (2005) Effects of strength training and nutritional counseling on metabolic health indicators in aging women. Canadian Journal of Applied Physiology 30: 690-707. 
26. Stewart KJ, Bacher AC, Turner KL, Fleg JL, Hees PS, et al. (2005) Effect of exercise on blood pressure in older persons: a randomized controlled trial. Archives of Internal Medicine 165: 756-762.

27. Taaffe DR, Galvao DA, Sharman JE, Coombes JS (2007) Reduced central blood pressure in older adults following progressive resistance training. Journal of Human Hypertension 21: 96-98.

28. Tsutsumi T, Don BM, Zaichkowsky LD, Delizonna LL (1997) Physical fitness and psychological benefits of strength training in community dwelling older adults. Applied Human Science 16: 257-266.

29. Terra DF, Mota MR, Rabelo HT, Bezerra LM, Lima RM, et al. (2008) Reduction of arterial pressure and double product at rest after resistance exercise training in elderly hypertensive women. Arquivos Brasileiros de Cardiologia 91: 299-305.

30. Thomas GN, Hong AW, Tomlinson B, Lau E, Lam CW, et al. (2005) Effects of Tai Chi and resistance training on cardiovascular risk factors in elderly Chinese subjects: a 12-month longitudinal, randomized, controlled intervention study. Clinical Endocrinology 63: 663-669.
31. Cononie C, Graves JE, Pollock ML, Phillips I, Summers C, et al. (1991) Effect of exercise training on blood pressure in 70-to 79yr-old men and women. Medicine \& Science in Sports \& Exercise 23: 505-511.

32. Dunstan DW, Daly RM, Owen N, Jolley D, De Courten M, et al. (2002) High-intensity resistance training improves glycemic control in older patients with type 2 diabetes. Diabetes Care 25: 1729-1736.

33. Wood RH, Reyes R, Welsch MA, Favaloro-Sabatier J, Sabatier M, et al. (2001) Concurrent cardiovascular and resistance training in healthy older adults. Medicine \& Science in Sports \& Exercise 33: 1751-1758.

34. Anton MM, Cortez-Cooper MY, DeVan AE, Neidre DB, Cook JN, et al. (2006) Resistance training increases basal limb blood flow and vascular conductance in aging humans. Journal of Applied Physiology 101: 1351-1355.

35. Carvalho Filho ET, Serro Azul LG, Curiate JAE (1983) Hipertensão arterial no idoso. Arquivos Brasileiros de Cardiologia 41: 211-220.

Copyright: (C2019 de Oliveira Jacques NM. This is an open-access article distributed under the terms of the Creative Commons Attribution License, which permits unrestricted use, distribution, and reproduction in any medium, provided the original author and source are credited. 